\title{
Study on the Migration of Silver Nanoparticles from Nano silver Food Packages into Food Liquid
}

\author{
Yang Yuhan ${ }^{1}$, Zhang Xiaoqing ${ }^{2}$, Li Hui ${ }^{1}$, Wang $\mathrm{He}^{1}$, Liu Ning ${ }^{1}$, Qiu Shi ${ }^{1}$, Bi Hongmei ${ }^{1 *}$
}

${ }^{1}$ College of Science, Heilongjiang Bayi Agricultural University, Daqing 163319, PR China

${ }^{2}$ Department of Finance and Property, Qingbei Service Company of Daqing oilfield, Daqing 163454, PR China

DOI: $10.36347 /$ sjavs.2021.v08i01.002

| Received: 22.12.2020 | Accepted: 08.01.2021 | Published: 11.01.2021

*Corresponding author: Bi Hongmei

\section{Abstract}

Original Research Article

The need for healthy food is much stringent than before. Food packaging is an important part in the production of food. The migration of silver nanopartilcles in nanosilver packages should be detected carefully. In this paper, the migration of silver nanoparticles in nano-silver/polypropylene milk storage packages was studied by UV-vis, which could be available in the evaluation of food packages.

Key words: Food package, Silver nanoparticles, food soup.

Copyright $(\mathcal{C} 2021$ The Author(s): This is an open-access article distributed under the terms of the Creative Commons Attribution 4.0 International License (CC BY-NC 4.0) which permits unrestricted use, distribution, and reproduction in any medium for non-commercial use provided the original author and source are credited.

\section{INTRODUCTION}

Nowadays, great attention has been paid to the health and safety of food. The desire for healthy food is much stronger than before. People are searching for natural, original, non-synthetic food. Nanotechnology is booming fast since $1990 \mathrm{~s}$, it has been integrated into the fields of medicine, biology, environment, energy, and agriculture and food science $[1,2]$. Food packaging is an important part in the conservation of food. Conventional packaging systems are designed to act as passive barriers against surrounding environment, which behave poorly in antimicrobial activity. Compared to conventional packaging, nanotechnologyenabled food packaging can be divided into two different key points: (i) improved packaging, where nanomaterials are mixed into the polymer matrix to improve the gas barrier properties such as polymer/clay nanocomposites; (ii) active packaging, where the nanoparticles interact directly with the food or environment to allow a better protection of the food [3].

Silver-based material is a kind of antibiotic with a broad spectrum of activity, which have also attracted great interest owing to its photoactivity of semiconductor photocatalysis, antibacterial activity and the presence of nanocrystallites [5-7]. While too much intake into body may have influence on the health of people, it is very important to detect the amount of silver nanopartilcles in food packages.

\section{MATERIALS AND METHODS \\ Materials and apparatus}

Four commercially available nanosilver/polypropylene milk storage packages were selected as research objects, and marked as A, B, C, D, respectively. White vinegar, plant Oil, milk, hot pot soup and deionized water were poured into four packages of A, B, C, D, respectively, and the packages were soaked for 60 days at room temperature. Every 5 days, the liquid samples of different packages were tested by UV-vis spectrometer (UV-2802PCS, UNICO).

\section{Migration of silver nanoparticles}

The migration situation of silver nanoparticles in packages was detected by UV-vis spectroscopy. The migration amount could be calculated by standard working curve of silver nanoparticles.

\section{RESULTS AND DISCUSSION}

Compared with deionized water, the UV spectra of white vinegar, plant oil, milk, hot pot soup were shown as follows. As shown in Fig.1, the migration of silver nanoparcles changed little in 60 days. And the differences of migration amount of the four nano-silver/polypropylene milk storage packages were little. 


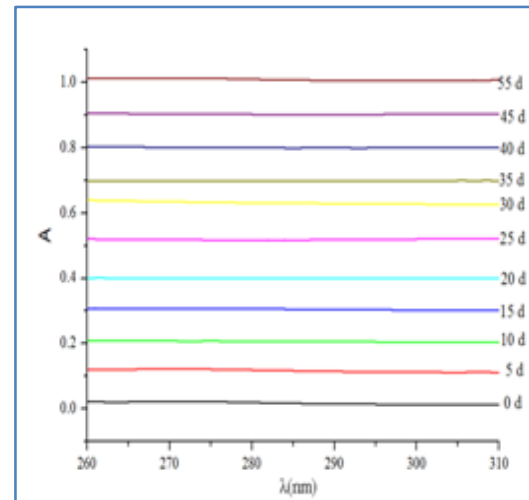

A

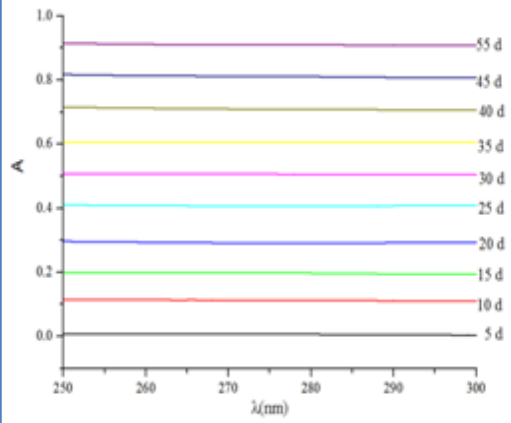

C

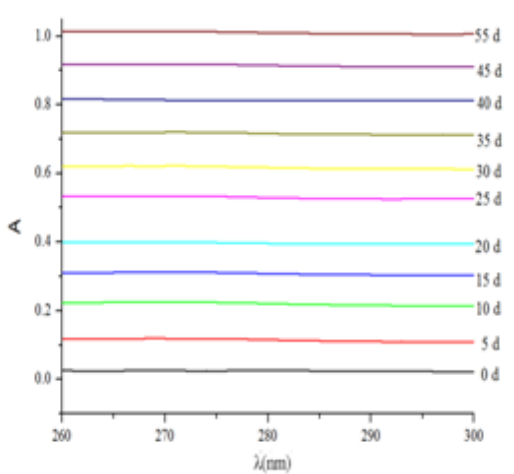

$\mathrm{B}$

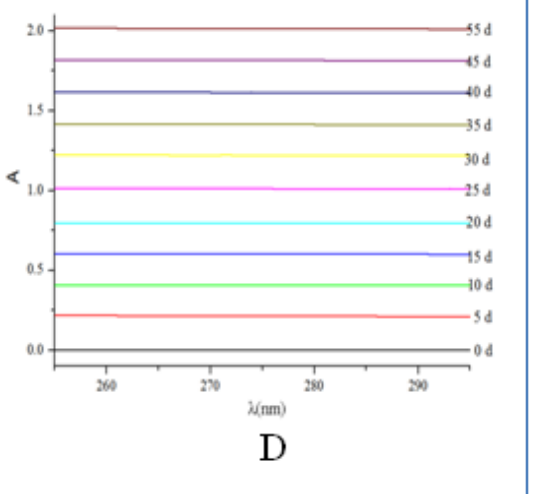

$\mathrm{D}$

Fig-1: Migration of nanosilver in different samples after soaking in deionized water

As we all know that the ingredients of hot pot soup were complex, the migration of silver nanoparticles changed obviously as the days went on as shown in Fig.2. It was shown that the migration of silver nanoparticles changed abnormally in the former 15 days, which might due to the decay of the hot pot soup. In a word, the migration of silver nanoparticles increased greatly in the food soup than in a simulated food liquid, and the migration amount is higher than that of in a simulated food liquid. The migration conditions of silver nanoparticles in four nanosilver/polypropylene milk storage packages were different, which might be attributed to the techniques by which the packages were made.

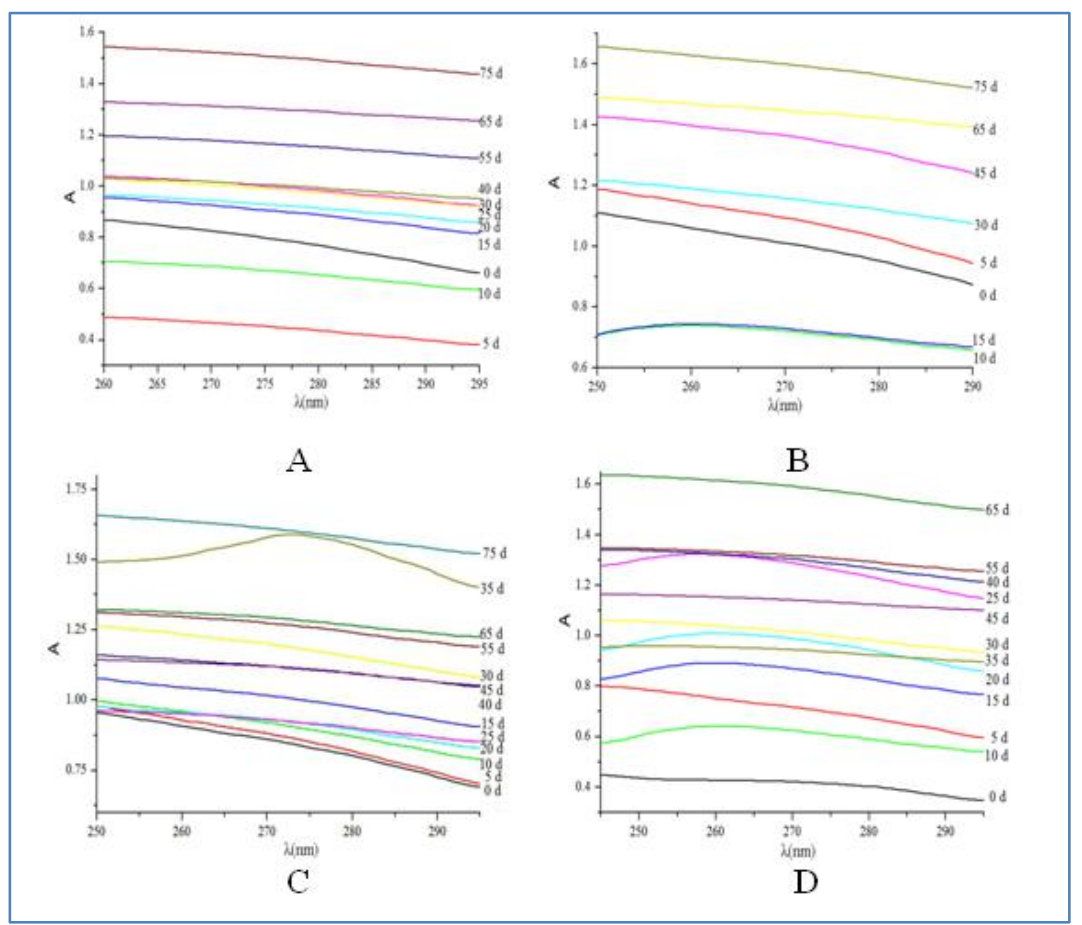

Fig-2: Migration of nanosilver in different samples after soaking in hot pot soup 


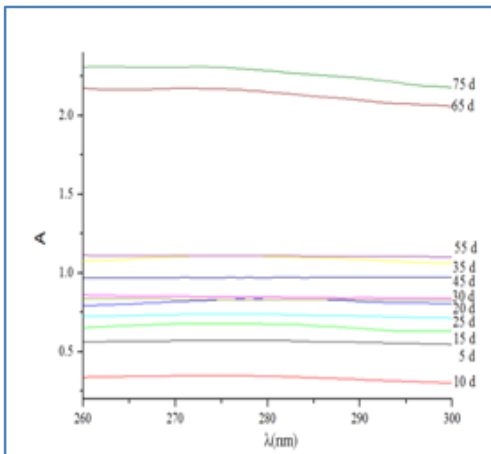

A

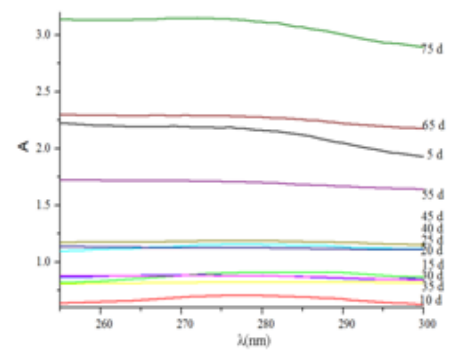

C

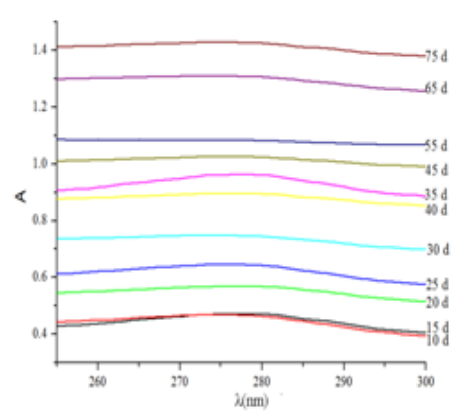

B

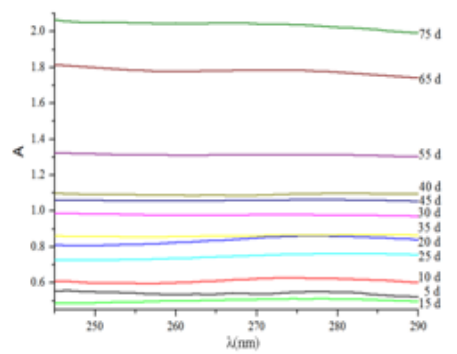

$\mathrm{D}$

Fig-3: Migration of nanosilver in different samples after soaking in milk

While soaked in milk, as shown in Fig.3, the migration of silver nanoparticles presented two stages. In the first stage, the migration amount of silver nanoparticles was low; in the second stage, the migration amount was higher. The migration rule of silver nanoparticles in four nano-silver/polypropylene milk storage packages were the same.

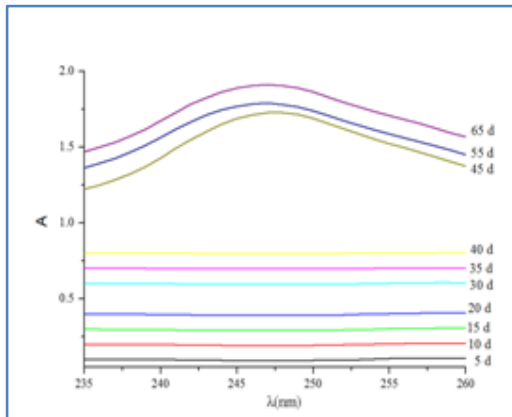

A

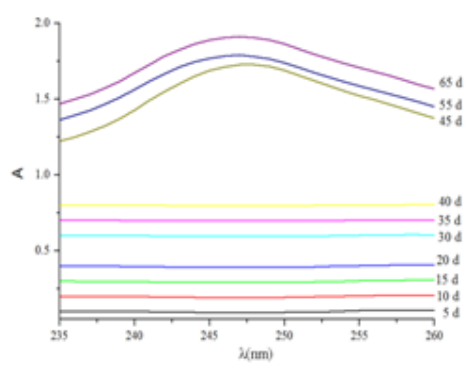

C

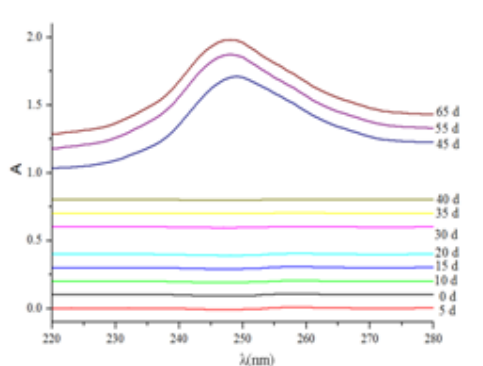

B

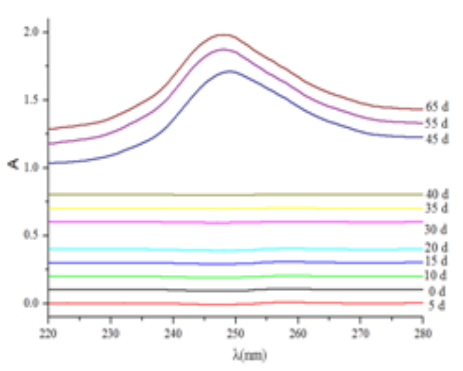

$\mathrm{D}$

Fig-4: Migration of nanosilver in different samples after soaking in white vinegar

While soaked in white vinegar, as shown in Fig.4, the migration amount of silver nanoparticles in four storage packages were very high, and also presented two stages. After soaking for 45 days, the absorption peak blue-shifted obviously. 


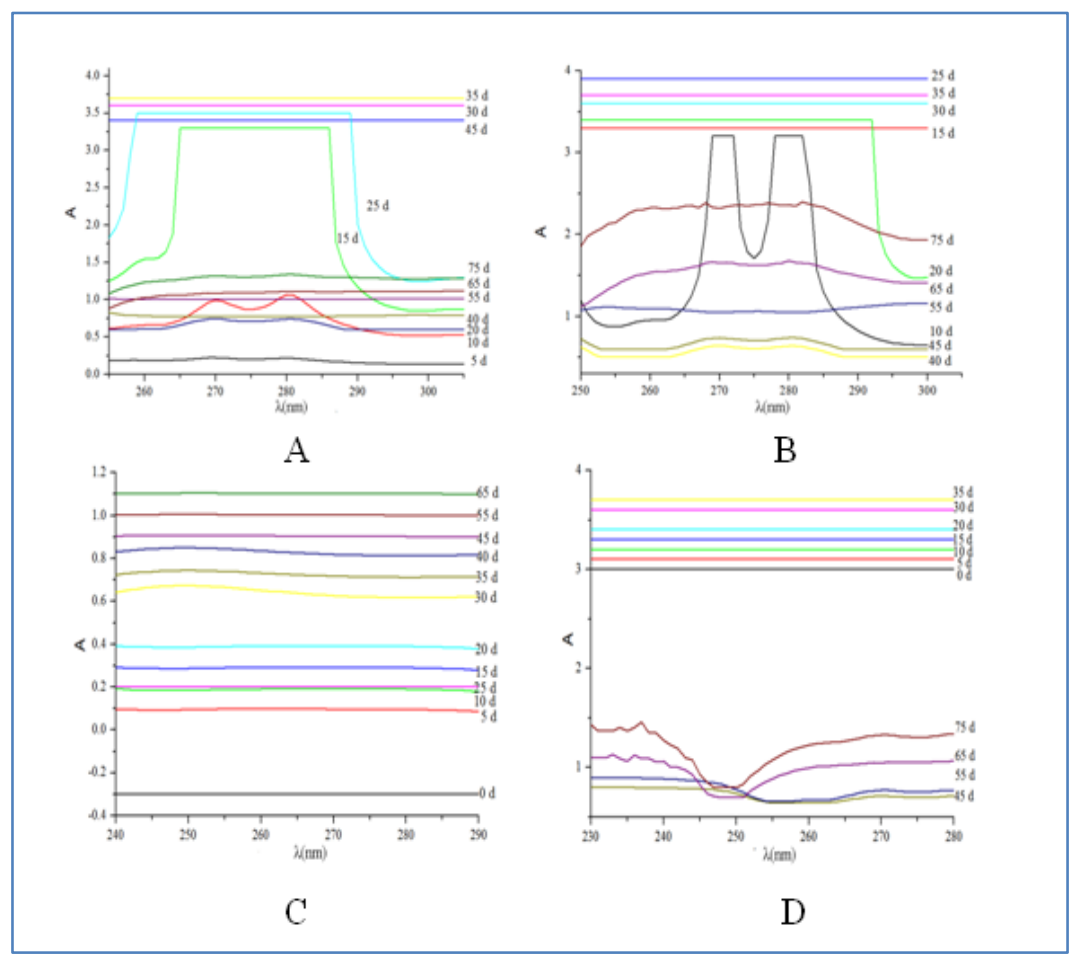

Fig-5: Migration of nanosilver in different samples after soaking in plant oil

While soaked in plant oil as shown in Fig.5, the migration of silver nanoparticles also presented two stages. In the former 35 days, the migration amount of silver nanoparticles was low; in the second stage, the migration conditions were more complicated. There were two adsorption peaks in A and B package, which might be the influence of parameter agent of cyclohexane [8].

\section{CONCLUSION}

In this paper, it was found that the migration of silver nanoparticles in food liquid are totally different compared with simulated liquids or chemical reagents. The result showed that the migration amount of silver nanoparticles was as follows: White vinegar $\approx$ Plant Oil $>$ Milk $>$ Hot Pot solution $>$ Water.

\section{ACKNOWLEDGEMENTS}

This work was supported by Undergraduate Training Program for Innovation and Entrepreneurship of Heilongjiang Province in China (No. 202010223076).

\section{REFERENCE}

1. Michael JP. Nanomaterial-the driving force. Materials Today. 2004; 7:20-29

2. Vance ME, Kuiken T, Vejerano EP. Nanoteachnology in the real world: Redeveloping the nanomaterial consumer products inventory.
2015; 6:1769-1780

3. Michael RT. Assuring the safety of nanomaterials in food packaging: the regulatory process and key issues. America: Project on Emerging Nanotechnologies. 1st. 2008.

4. Piccinno F, Gottschalk F, Seeger S. Industrial production quantities and uses of ten engineered nanometerials in Europe and the world. Journal of Nanoparticle Research. 2012; 14(9):1-11.

5. Marilena Carbone, Domenica Tommasa Donia, Gianfranco Sabbatella, Riccarda Antiochia.Silver nanoparticles in polymeric matrices for fresh food packaging. Journal of King Saud UniversityScience. 2016; 28(4):273-279.

6. Li Li, Chanjuan Zhao, Yadong Zhang, Jianfeng Yao, Wenjian Yang, Qiuhui Hu, Cailin Wang, Chongjiang Co. Effect of stable antimicrobia nanosilver packaging on inhibiting mildew and instorage of rice. Food Chemistry. 2017; 215:477482.

7. Jokar M, Abdul Rahman R, Ibrahim N. Melt production and antimicrobial efficiency of LowDensity polyethylene (LDPE)-Silver nanocomposite film. 2012; 5(2): 719-728.

8. Xue-lin Tian, Wei-hua Wang, Kai Chen, etc. Growth and Stabilization of Silver Nanoplates in Aqueous Solvent Monitored Though UV-Vis Spectra [J]. Chinese Journal of Chemical Physics. 2006, 19(4): 362-365. 